\title{
Spectrum of diseases presenting with lymphadenopathy in elderly: Fine needle aspiration cytology of 60 cases
}

\author{
Anirudha V Kushtagi ${ }^{1}$, Kazi Wajid Husain ${ }^{2, *}$, Vinay Kumar $\mathbf{R}^{3}$ \\ ${ }^{\mathbf{1}}$ Associate Professor, ${ }^{\mathbf{2 , 3}}$ Assistant Professor, ${ }^{\mathbf{1 - 3}}$ Dept. of Pathology, Koppal Institute of Medical Sciences
} Koppal, Karnataka India

*Corresponding Author:

Email: go4kazi@gmail.com

\begin{abstract}
Introduction: Lymphadenopathy is one of the common clinical presentations in patients of all age group. Lymphadenopathy can be due to reactive or infective cause, but malignancy including metastastic deposits in are not uncommon in elderly. Fine needle aspiration cytology (FNAC) is a useful diagnostic rapider in many mass lesions like lymphadenopathy.

Aims and Objectives: Three years study was conducted to know the spectrum of diseases causing enlargement of lymph nodes in elderly patients with age 60 years and above.

Materials and Methods: We have collected all the clinical data from the registers from June 2014 to June 2017 in Medical records section, KIMS, Koppal and reviewed FNAC slides of lymph node aspirates of elderly.

Results: A total of 60 elderly patients of age 60 years and above with lymphadenopathy were included in this study. Cervical group of lymph nodes were the predominant site for lymphadenopathy. The commonest cause of lymphadenopathy was reactive, seen in 29 patients, followed by 23 patients with metastatic deposits and squamous cell differentiation was commonest type. Tubercular and suppurative lymphadenitis were observed in four cases each. CBNNAT is an useful diagnostic modality in microbiological confirmation of tuberculosis. Males showed preponderance of reactive as well as in metastatic lymphadenopathy. Conclusion: Though reactive lymphadenitis is the commonest cause for enlargement of lymph nodes but metastatic deposits are not uncommon in elderly. FNAC as a diagnostic rapider useful screening tool for all elderly patients presenting with lymphadenopathy.
\end{abstract}

Keywords: Lymphadenopathy, Reactive, Metastatic, FNAC, Tubercular, CBNAAT.

\section{Introduction}

Lymphadenopathy is one of the common clinical presentations in patients of all age group, the etiology range from nonspecific reactive, inflammation, lymphoproliferative disease to metastatic deposits. ${ }^{1,2}$

In 1904 Greig \& Gray first time aspirated the Lymph node aspiration to diagnose trypanosomiasis. Gutherie in 1921 tried to correlate various diseases with Lymph node aspirate cytology. ${ }^{2,3}$ In last few decades Fine Needle Aspiration Cytology (FNAC) become an important, reliable, SAFE, first line diagnostic tool. SAFE (Simple Accurate Fast \& Economical) an attractive abbreviation introduced for FNAC by DeMay. ${ }^{4}$

Probably due to the proximity of cervical lymph nodes to anatomical passages like aero-digestive tract which are being exposed frequently to environmental infectious agents. Cervical group of lymph nodes drain major part of the body from head and neck, thorax and few abdominal organs which explains the cervical group is the most common lymph node group which present with enlargement in many medical conditions from reactive to metastatic deposits. ${ }^{4}$

The ethology of lymphadenopathy in elderly is likely to be different in contrast to children as well as from young adults. In children and young adults reactive lymphadenopathy is more common and metastatic deposits in elderly are not uncommon. ${ }^{2}$
FNAC as a sensitive reliable diagnostic tool and it's turnaround time for the diagnosis of lesions is lesser than the histopathological study. ${ }^{1}$

According our search, there are studies available in the literature about FNAC and lymphadenopathy in various age groups but sparse studies are available pertaining to role of FNAC in elderly patients presenting with lymphadenopathy. Present retrospective study emphasizes the various causes of lymphadenopathy in elderly patients with age 60 years and above.

\section{Materials and Methods}

Present three year retrospective study was conducted at Koppal institute of medical sciences Koppal (KIMS), Karnataka, India.

This three years study was conducted to know the spectrum of diseases causing enlargement of lymph nodes in elderly patients with age 60 years and above, also to know the incidence of infective and malignant etiology including metastatic involvement of lymph and also to know the spectrum of diseases causing lymphadenopathy in elderly females and elderly males.

We collected all the clinical data from the registers between June 2014 to May 2017 in Medical records section, KIMS, Koppal. We also reviewed FNAC slides of Lymph node aspirates which were stored in our department and re-examined them under microscope of all elderly registered patients. When we searched the 
records, most of the reports of FNA aspirates were reported on the same day, indicating it as a diagnostic rapider.

Also we have considered the lymph node FNAC slides received for second opinion of elderly patients from private laboratories in and around Koppal.

The slides which we have re-examined were stained by Geimsa, fields, haematoxylene \& eosin, papenicolous as well as by Zeel-Neelson for acid fast bacilli in suspected cases of tubercular lymphadenopathy. All the FNACs were done by the 2224 gauge needles on 5-10 ml syringes. Aspirate from one elderly patient with presumptive tubercular lymphadenitis, subjected to cartridge based nucleic acid amplification test available at our District Hospital KIMS, Koppal.

\section{Results}

A total of 60 elderly patients with age of 60 years and above who presented with enlarged lymph nodes of various sites were included in this study. The commonest cause of lymphadenopathy was reactive and seen in 29 patients, followed by malignant etiology in 23 patients, metastatic deposits with suspected primary in the upper aero-digestive tract in 22 , as patients presented with cervical lymphadenopathy and one patient with axillary lymphadenopathy had features of adencarcinoma and the patient was elderly female presented with breast lump. Four cases were diagnosed as Kochs (granulomatous/abscess) disease and acid fast bacilli was confirmed in one patient. Suppurative lymphadenopathy was seen in four patients. Aaspirate of two elderly patients was inadequate for opinion as aspirate revealed only haemorrhagic aspirate. Amongst four tubercular lymphadenopathy patients, one elderly female presented with supraclavicular lymphadenopathy and reported as suppurative lesion with suspected tubercular abscess on FNAC, but acid fast bacilli was not detected in the aspirate on Zeelneelson stain preparation, hence reaspiration done and subjected in to Cartidge Based Nucleic Acid Amplification Test (CBNAAT) available at our teaching and district hospital Koppal and was detected refampicin sensitive mycobactrium tuberculosis. Followung table showing distribution of pathological conditions in each lymph node groups (Table 1).

Table 1: Distribution pathological conditions in each lymph node groups

\begin{tabular}{|l|c|c|c|c|}
\hline $\begin{array}{c}\text { Lymph Node Group } \\
\text { Involved }\end{array}$ & Reactive & Granulomatous & Suppurative & Malignant/Metastatic \\
\hline Cervical (46) & 20 & 02 & 02 & 22 \\
\hline Axillary (05) & 02 & 01 & 01 & 01 \\
\hline Inguinal (05) & 05 & 00 & 00 & 00 \\
\hline Supraclavicular (04) & 02 & 01 & 01 & 00 \\
\hline Total (60) & 29 & 04 & 04 & 23 \\
\hline
\end{tabular}

23 patients were diagnosed as metastatic deposits out of which 17 cases were diagnosed as metastatic deposits with squamous cell differentiation, 03 patients were diagnosed as deposits with features of poorly differentiated carcinoma and 02 female patients, one with cervical and other with axillary lymphadenopathy presented features of adenocarcinoma on aspirates. One patient was presented with atypical cell suspected deposits with squamous cell differentiation Out of 23 cases with metastatic deposits, 19 patients were males and 04 were females.

In the present study, lymphadenopathy is most commonly seen in Males and included 43 patients, whereas only 17 Female patients presented with lymphadenopathy. The male to female ratio obtained in our study is $2.5: 1$. The cause of lymphadenopathy in each gender is enlisted in the following table.

Table 2: Gender wise distribution of pathological conditions causing lymphadenopathy in elderly

\begin{tabular}{|l|c|c|}
\hline \multicolumn{1}{|c|}{ Cause of Lymphadenopathy } & In Males & In Females \\
\hline Reactive & 22 & 06 \\
\hline Metastatic & 19 & 04 \\
\hline Tubercular & 01 & 03 \\
\hline Suppurative & 01 & 03 \\
\hline Total & 43 & 17 \\
\hline
\end{tabular}

Analysis of gender distribution of etiology of lymphadenopathy revealed ractive and metastatic deposits were more common in males when compared with females whereas Infective pathology like tubercular and suppurrative lymphadenitis were detected commonly in females as mentioned in the above table (Table 2). 


\section{Discussion}

In patients with lymphadenopathy, FNAC has a significant role in categorizing the lesions to reactive, inflammatory, infectious, primary malignant condition like lymphoma or secondary metastatic deposits. In patients with metastatic lymphadenopathy of unknown primary, FNAC of such enlarged lymph nodes not only confirms metastatic deposits but also may give a clue regarding primary site of malignancy and helps in further management. ${ }^{1}$

In our study the most common lymph node group involved was cervical and common finding was reactive lymphadenopathy followed by metastatic deposits. The most common malignancy metastasizing to lymph nodes was deposits of squamous cell carcinoma and probably most of them were from upper aero-digestive tract. These findings correlated well with other previous studies in literature search. ${ }^{1-4}$

According to studies available in the literature the most common age group for metastatic lymphadenopathy, ranged between 50 to 80 Years.

FNAC as a sensitive tool helps us to reduce the turnaround time necessary for the diagnosis of Lesions and further management in comparision to histopathological study. However biopsy study remains the diagnostic. 1 FNAC is a diagnostic rapider tool.

According to Baji S N et al study, incidence of reactive lymphadenitis showed a downward trend in sixth decade and malignant lesions took over. The authors also explained that the need of fnac in lymphadenopathy is significant in the elderly, which determines the clue to diagnosis. ${ }^{1}$

FNAC is of considerable value in disease staging and documentation of metastasis in known primary and occult tumors. Common metastatic tumors to lymph nodes include malignancies of thyroid, respiratory system, gastro intestinal system, male and female genital tracts.

In study of Ghartimagar D et.al, 93 lymph nodes were reported as positive for malignancy. Cervical lymph nodes were the most common site for metastatic deposits. The highest incidence of metastatic deposits were seen in patients with age of 60 years and above with $57(61 \%)$ of cases. In their study, adeno carcinoma was the commonest type of metastatic deposits followed by squamous cell carcinoma. Incidence of metastatic deposits was more common in females. Our findings correlated with this study in relation to the site and age. Where as in contrast males were commonly affected and squamous cell carcinoma was the commonest type in our study similar to the findings of study by Mainali N and Suwal R B and other by Mohanty R and Wilkinson A. ${ }^{5-7}$

In a study by Tandon P and Gautam W, 44 cases were studied and they were above 60 years of age and presented with different groups of enlarged lymph nodes. 24 were metastatic, 9 reactive, 4 suppurative and
3 cases were tubercular lymphadenitis. These findings were similar to our study findings. ${ }^{4}$

The frequent involvement of cervical lymph nodes in disease process is possibly due to their proximity to anatomical passages of body like upper aero digestive tract and head and neck structures. They drain lymphatics from major part of the body. They are not only involved in infectious pathology but also in metastastic deposits from primary sites of malignancy. ${ }^{4}$

In our study cervical lymph nodes were the commonest site for metastasis and Sqamous cell differentiation being the most common type. Similar findings were seen in the study articles by Mainali $\mathrm{N}$ and Suwal R B, Dr Ripunjaya Mohonty and Dr Anne Wilkinson. ${ }^{6,7}$ According to the study by Naresh N Rai, FNAC is an useful diagnostic tool in detecting metastatic deposits in lymph nodes which provides clue for the primary lesion and further management of the condition. In their study most common metastatic deposit was squamous cell carcinoma which was similar to many studies ${ }^{9}$ and also comparable with our study. ${ }^{8}$ Metastastic deposits were the common cause of lymphadenopathy than primary Lymphomas in elderly people Gupta R and Dewan), ${ }^{9}$ in our study we have not reported any primary lymphoma case.

Similar to our study, Khajuria $\mathrm{R}$ et.al, showed Cervical lymph nodes were the most common groups which clinically presented with enlargement. Reactive Lymphadenitis was the commonest type and out of 25 metastatic deposits, squamous cell carcinoma was the most common type. ${ }^{10}$

Histopathology remains the corner stone of the diagnosis, but in infectious conditions like tubercular lymphadenopathy FNAC suffices without the need of more invasive procedure. Diagnosis can be made confidently with additional tools which include the basic Zheil Neelson stain for demonstration of acid fast bacilli and the more advanced Cartridge bases nucleic acid amplification test which rules out refampicin resistant or sensitive tuberculosis. The combined utility of FNAC, ZN staining and CBNAAT acts as an effective tool in the diagnosis of presumptive TB lymph node.

CB-NAAT as a rapid diagnostic test, should be routinely used in public and private health sectors efficiently for the detection of a case of tuberculosis. ${ }^{11}$

In cases of extrapulmonary tuberculosis, nucleic acid amplification tests appears to be an attractive modality with a high specificity, positive predictive value and highly variable sensitivity. ${ }^{12}$

\section{Conclusion}

Time and again proves, FNAC is a sensitive and diagnostic rapider in patients presenting with lymphadenopathy, it helps us to reduce the turnaround time needed for the diagnosis of lesions. It is useful in differentiating from infective to malignant etiology including metastatic deposits and further management. 
In our study cervical group of lymph nodes was the commonest site obsereved with reactive followed by metastatic etiology. Male preponderance was noted in reactive as well as in metastatic deposits; the frequent finding of metastatic deposits was of squamous cell differentiation.

Though reactive lymphadenitis is the commonest cause for enlargement of lymph nodes but metastatic deposits are not uncommon in elderly. Hence FNAC can be used as screening tool for all elderly patients presenting with lymphadenopathy.

Infective including Tubercular etiology was observed female preponderance. The combined utility of FNAC, ZN staining and CBNAAT acts as an effective tool in the diagnosis of presumptive or suspected TB lymph node with granulomas, necrosis or abscess.

Acknowledgements: We would like to thank Dr Shankar M Malapure, Director, KIMS, Koppal for his encouragement in preparing this article. Authors would like to thank Dr Ajay Shrof, Asst. Professor, FM, KIMS, Koppal for his help during preparation of this article and to all the staff members of the Department of Pathology, KIMS, Koppal. We would also like to thank Dr Ramesh G Moolimani, DTO, Koppal, for his valuable support. We thank all our family members, all the patients and almighty.

\section{References}

1. Baji SN, Anand V, Sharma R, Deore KS, Chokshi M. Analysis of FNAC of cervical lymph nodes: Experience over a two years period. Int J Med Sci Public Health 2014;3(5):607-9.

2. Agarwal D, Bansal P, Rani B, Sharma S, Chawla S, Bharath V et al. Evaluation of etiology of lymphadenopathy in different age groups using fine needle aspiration cytology: A retrospective study. The Internet Journal of Pathology 2009;10(2):12-20.

3. Amit KN, Kirti N, Jha J, Sudhir K. Role of fine needle aspiration cytology in assessment of cervical lymphadenopathy in variable age groups: a retrospective study. Int J Med Res Health Sci. 2016;5(11):306-10.

4. Tandon P, Gautam W. Utility of fine needle aspiration, Utility of Fine Needle Aspiration Cytology in Lymphadenopathy - A Study of 638 Cases in a Primary Care Setting. NJLM 2016;5(3):11-5.

5. Ghartimagar D, Ghosh A, Ranabhat S, Shrestha MK, Narasimhan R, Talwar OP. Utility of fine needle aspiration cytology in metastatic lymph nodes. Journal of Pathology of Nepal 2011;1(2):92-5.

6. Mainali N, Suwal RB. Patterns of lymphadenopathy on fine needle aspiration cytology in eastern Nepal. Journal of Pathology of Nepal 2015;5(10):814-6.

7. Mohanty R, Wilkinson A. Utility of Fine Needle Aspiration Cytology of Lymph nodes. IOSR Journal of Dental and Medical Sciences 2013;8(5):13-8.

8. Rai NN, Patangia P, Meena SP. Role of fine needle aspiration cytology in diagnosis of metastatic lymphadenopathy. J. Evid. Based Med. Healthc. 2016;3(18):738-41.

9. Gupta R, Dewan D. Etiological pattern of lymphadenopathies and role of fine needle aspiration cytology (FNAC) in its diagnosis. IMJH 2015;1(8):12-9.

10. Khajuria R, Goswami KC, Singh K, Dubey VK. Pattern of Lymphadenopathy on Fine Needle Aspiration cytology in Jammu. JK Science: Journal of Medical Education and Research 2006;8 (3):157-9.

11. Kandi S, Reddy V, Nagaraja SB. Diagnosis of pulmonary and extra pulmonary tuberculosis: How best is CBNAAT when compared to conventional methods of TB detection? Pulm Res Respir Med Open J. 2017;4(2):3841.

12. Fuladi AB, Gupta PP. Challenges in the Diagnosis of Extrapulmonary Tuberculosis: Role of Gene Xpert Mycobacterium Tuberculosis/Rifampicin Assay. Int J Sci Stud 2017;5(7):75-79. 